Proc. Indian Acad. Sci. (Math. Sci.), Vol. 106, No. 3, August 1996, pp. 261-270.

(C) Printed in India

\title{
Solution of singular integral equations with logarithmic and Cauchy kernels
}

\author{
A CHAKRABARTI and T SAHOO \\ Department of Mathematics, Indian Institute of Science, Bangalore 560012, India
}

MS received 23 January 1996; revised 20 April 1996

\begin{abstract}
A direct method of solution is presented for singular integral equations of the first kind, involving the combination of a logarithmic and a Cauchy type singularity. Two typical cases are considered, in one of which the range of integration is a single finite interval and, in the other, the range of integration is a union of disjoint finite intervals. More such general equations associated with a finite number (greater than two) of finite, disjoint, intervals can also be handled by the technique employed here.
\end{abstract}

Keywords. Water waves; scattering; Cauchy kernel; logarithmic kernel.

\section{Introduction}

The singular integral equation

$$
\begin{aligned}
& \int_{L .} f(t)\left[K \ln \left|\frac{x-t}{x+t}\right|+\frac{1}{x+t}+\frac{1}{x-t}\right] \mathrm{d} t=g(x), \quad x \in L, \\
&(K>0, \text { a known constant })
\end{aligned}
$$

with $L$ representing either (i) a single finite interval $(a, b)$, or (ii) a union of two disjoint finite intervals of the type $(a, b) \cup(c, d)$, where $0<a<b<c<d$, has been considered for solution by employing a direct method which reduces such integral equations to an equation having only a Cauchy type singularity.

Singular integral equations of the form (1.1), where the unknown function $f(x)$ possesses square-root singularities at the end points of $L$ (see Mandal [3]) occur in the study of scattering and radiation of surface water waves, in the linearized theory, by vertical barriers possessing either (i) a single gap, in the case when $L=(a, b)$ or (ii) two gaps, in the case when $L=(a, b) \cup(c, d)$. Recently, Banerjea and Mandal [6] have presented a method of solving eq. (1.1) when $L=(a, b)$, by reducing it to a singular integral equation over two disjoint intervals, with a Cauchy type singularity, and employing a function theoretic approach to the resulting equation. Banerjea and Mandal [6] have also mentioned that eq. (1.1) cannot be reduced to an equation with a Cauchy-type singularity, of a type which has been possible to equations of this form, (see Ursell [2]) where $L$ represents a single interval of the form $(0, a)$ or $(b, \infty)$, in which case, the end behaviours of the unknown function $f(x)$ have a different structure as compared to the ones required for (1.1).

In the present paper, we have shown that a method, similar to Ursell's [2] is applicable to the integral equation (1.1), which reduces the equation to the one which involves only a Cauchy type singularity and which can be solved by using well-known results available in the books of Muskhelishvili [1] and Gakhov [4], as well as in a recently published paper of Chakrabarti and George [5]. 
The all-important identity that has helped in arriving at the closed form solution of the integral equation (1.1) by our approach is the one as given by the relation

$$
\ln \left|\frac{x-\alpha}{x+\alpha}\right|=2 x \int_{0}^{\alpha} \frac{\mathrm{d} t}{t^{2}-x^{2}}, \quad 0<x<\alpha .
$$

In the next section, we take up the solution of (1.1) in the two cases (i) and (ii) described above, and present the solutions in closed forms.

\section{Methods of solutions}

Case (i). $L=(a, b)$

In this case, we look for the solution of (1.1) along with the end point requirements

$$
f(x) \approx\left\{\begin{array}{lll}
O\left(|x-a|^{-1 / 2}\right) & \text { as } & x \rightarrow a, \\
O\left(|x-b|^{-1 / 2}\right) & \text { as } & x \rightarrow b .
\end{array}\right.
$$

We set, as in the work of Ursell [2],

so that

$$
F(x)=\int_{x}^{b} f(t) \mathrm{d} t \text { for } a<x<b
$$

$$
F(b)=0 \quad \text { and } \quad F^{\prime}(x)=-f(x) \text { for } a<x<b .
$$

Substituting for $f(x)$ from (2.3) in (1.1), we obtain the singular integral equation

where

$$
\int_{a}^{b} \frac{\lambda(t) \mathrm{d} t}{t^{2}-x^{2}}=h(x) \text { for } a<x<b
$$

and

$$
\begin{aligned}
& \lambda(x)=F^{\prime}(x)+K F(x) \text { for } a<x<b, \text { so that } \\
& f(x)=\frac{\mathrm{d}}{\mathrm{d} x}\left[\mathrm{e}^{-K x} \int_{x}^{b} \hat{\lambda}(u) \mathrm{e}^{K u} \mathrm{~d} u\right] \text { for } a<x<b
\end{aligned}
$$

with

$$
h(x)=\frac{1}{2 x}\left\{g(x)-K F(a) \ln \left|\frac{x-a}{x+a}\right|\right\} \quad \text { for } a<x<b
$$

$$
\lambda(x) \approx\left\{\begin{array}{lll}
O\left(|x-a|^{-1 / 2}\right) & \text { as } & x \rightarrow a \\
O\left(|x-b|^{-1 / 2}\right) & \text { as } & x \rightarrow b
\end{array}\right.
$$

The solution of the integral equation (2.4) is well-known and is given by (see Gakhov [4])

where

$$
\lambda(x)=\frac{2 C x}{R(x)}-\frac{4 x}{\pi^{2}} \int_{a}^{b} \frac{t R(t) h(t) \mathrm{d} t}{R(x)\left(t^{2}-x^{2}\right)} \text { for } a<x<b,
$$

$$
R(t)=\sqrt{\left(t^{2}-a^{2}\right)\left(b^{2}-t^{2}\right)} \text { for } a<t<b,
$$

and $C$ is an arbitrary constant.

We next mention the following results to be obtained by standard methods (see Gakhov [4] problem 18, pp. 81)

$$
\int_{a}^{b} \frac{R(t) g(t) \mathrm{d} t}{R(x)\left(t^{2}-x^{2}\right)}=\frac{C_{2}}{R(x)}+\Delta(x) \int_{a}^{b} \frac{1}{\Delta(t)} \frac{g(t) \mathrm{d} t}{t^{2}-x^{2}} \text { for } a<x<b,
$$


where

$$
\begin{aligned}
& C_{2}=\int_{a}^{b} \frac{g(t)}{\Delta(t)} \mathrm{d} t, \Delta(x)=\sqrt{\frac{x^{2}-a^{2}}{b^{2}-x^{2}}} \text { for } a<x<b, \\
& \ln \left|\frac{t-a}{t+a}\right|=2 t \int_{0}^{c} \frac{\mathrm{d} \xi}{\xi^{2}-t^{2}} \text { for } 0<t<a, \\
& \int_{a}^{b} \frac{t R(t) \mathrm{d} t}{t^{2}-u^{2}}=\frac{\pi}{2}\left[\frac{a^{2}+b^{2}}{2}-u^{2}\right] \text { for }(a<u<b), \quad \text { (R3) } \\
& \int_{a}^{b} \frac{t R(t) \mathrm{d} t}{t^{2}-u^{2}}=\pi\left\{-\sqrt{\left(a^{2}-u^{2}\right)\left(b^{2}-u^{2}\right)}-u^{2}+\frac{a^{2}+b^{2}}{2}\right\} \text { for } \quad(0<u<a), \\
& \int_{a}^{b} R(t) \ln \left|\frac{t-a}{t+a}\right| \frac{\mathrm{d} t}{t^{2}-u^{2}}=\pi a+\pi \int_{0}^{a} \frac{\sqrt{\left(a^{2}-t^{2}\right)\left(b^{2}-t^{2}\right)} \mathrm{d} t}{t^{2}-u^{2}} \text { for } a<u<b
\end{aligned}
$$

Using the above mentioned results (R1) to (R5), from the relation (2.8) we obtain

where

$$
\begin{aligned}
\lambda(x)= & \frac{x}{R(x)}\left[Q+\frac{2 K}{\pi} F(a) G(a, b, x)\right] \\
& -\frac{2 x}{\pi^{2}} \Delta(x) \int_{a}^{b} \frac{1}{\Delta(t)} \frac{g(t) \mathrm{d} t}{t^{2}-x^{2}} \text { for } a<x<b,
\end{aligned}
$$

$$
\begin{aligned}
Q & =2\left[C-\frac{C_{2}}{\pi^{2}}+\frac{K a F(a)}{\pi}\right] \text { and } \\
G(a, b, x) & =\int_{0}^{a} \frac{\sqrt{\left(a^{2}-\xi^{2}\right)\left(b^{2}-\xi^{2}\right)} \mathrm{d} \xi}{\xi^{2}-x^{2}}, \quad a<x<b .
\end{aligned}
$$

From the relations (2.2), (2.5) and the expression (2.10), we obtain the relation connecting the constants $Q$ and $F(a)$ as given by

$$
\mathrm{e}^{K a} F(a)=\int_{a}^{b} u \mathrm{e}^{K u}\left(\frac{[Q+(2 K / \pi) F(a) G(a, b, u)]}{R(u)}-\frac{2}{\pi^{2}} \Delta(u) \int_{a}^{b} \frac{1}{\Delta(t)} \frac{g(t) \mathrm{d} t}{t^{2}-u^{2}}\right) \mathrm{d} u
$$

Also, from relations (2.5) and (2.10) we finally obtain the complete solution of (1.1) in the case when $L=(a, b)$, as given by

where

$$
\begin{aligned}
f(x)=\frac{\mathrm{d}}{\mathrm{d} x} & \left\{\mathrm { e } ^ { - K x } \int _ { b } ^ { x } u \mathrm { e } ^ { K u } \left(\frac{[\hat{Q}-(2 K / \pi) F(a) G(a, b, u)]}{R(u)}\right.\right. \\
& \left.\left.+\frac{2}{\pi^{2}} \Delta(u) \int_{a}^{b} \frac{1}{\Delta(t)} \frac{g(t) \mathrm{d} t}{t^{2}-u^{2}}\right) \mathrm{~d} u\right\} \text { for } a<x<b,
\end{aligned}
$$

$$
\hat{Q}=-Q
$$

The solution (2.13) agrees, in principle, with the one obtained by Banerjea and Mandal [6] recently. 
The solution of (1.1) can also be obtained, in this case, by setting

$$
\tilde{F}(x)=\int_{a}^{x} f(t) \mathrm{d} t \text { for } a<x<b .
$$

Proceeding in a similar manner as discussed above, we find the following alternative form of the solution $f(x)$ as given by

$$
\begin{aligned}
f(x)=\frac{\mathrm{d}}{\mathrm{d} x}\left\{\mathrm { e } ^ { - K x } \int _ { a } ^ { x } u \mathrm { e } ^ { K u } \left(\frac{[\tilde{Q}-(2 K / \pi) \tilde{F}(b) G(a, b, u)]}{R(u)}\right.\right. \\
\left.\left.+\frac{2}{\pi^{2}} \Delta(u) \int_{a}^{b} \frac{1}{\Delta(t)} \frac{g(t) \mathrm{d} t}{t^{2}-u^{2}}\right) \mathrm{~d} u\right\} \text { for } a<x<b,
\end{aligned}
$$

where the constants $\tilde{Q}$ and $\tilde{F}(b)$ are related as

$$
\mathrm{e}^{K a} \tilde{F}(b)=\int_{a}^{b} u \mathrm{e}^{K u}\left(\frac{[\tilde{Q}-(2 K / \pi) \tilde{F}(b) G(a, b, u)]}{R(u)}+\frac{2}{\pi^{2}} \Delta(u) \int_{a}^{b} \frac{1}{\Delta(t)} \frac{g(t) \mathrm{d} t}{t^{2}-u^{2}}\right) \mathrm{d} u .
$$

Next, we will discuss the method of solution of (1.1) when $L$ is the union of two disjoint finite intervals.

Case (ii). $L=(a, b) \cup(c, d)$

In this case, we desire to solve (1.1) where

$$
\begin{aligned}
& O\left(|x-a|^{-1 / 2}\right) \text { as } x \rightarrow a, \\
& O\left(|x-b|^{-1 / 2}\right) \text { as } x \rightarrow b, \\
& f(x) \approx O\left(|x-c|^{-1 / 2}\right) \text { as } x \rightarrow c \text {, } \\
& O\left(|x-d|^{-1 / 2}\right) \text { as } x \rightarrow d .
\end{aligned}
$$

We set

$$
f(x)=\left\{\begin{array}{lll}
f_{1}(x) & \text { for } & x \in(a, b) \\
f_{2}(x) & \text { for } & x \in(c, d)
\end{array}\right.
$$

and

$$
g(x)=\left\{\begin{array}{lll}
g_{1}(x) & \text { for } & x \in(a, b) \\
g_{2}(x) & \text { for } & x \in(c, d)
\end{array}\right.
$$

Then, defining

so that

$$
F_{1}(x)=\int_{x}^{b} f_{1}(t) \mathrm{d} t \text { for } a<x<b
$$

and

$$
\begin{aligned}
& F_{1}^{\prime}(x)=-f_{1}(x) \text { for } a<x<b \\
& F_{1}(b)=0
\end{aligned}
$$

$$
F_{2}(x)=\int_{x}^{d} f_{2}(t) \mathrm{d} t \text { for } c<x<d,
$$

so that

$$
\begin{aligned}
& F_{2}^{\prime}(x)=-f_{2}(x) \text { for } c<x<d \text { and } \\
& F_{2}(d)=0
\end{aligned}
$$


We reduce (1.1) into the following new equation

where

$$
\int_{a}^{b} \frac{\lambda_{1}(t) \mathrm{d} t}{t^{2}-x^{2}}+\int_{c}^{d} \frac{\hat{\lambda}_{2}(t) \mathrm{d} t}{t^{2}-x^{2}}=\frac{\tilde{g}(x)}{2 x} \text { for } x \in(a, b) \cup(c, d),
$$

and

$$
\begin{aligned}
& \lambda_{1}(x)=F_{1}^{\prime}(x)+K F_{1}(x) \text { for } a<x<b \text { and } \\
& \hat{\lambda}_{2}(x)=F_{2}^{\prime}(x)+K F_{2}(x) \text { for } c<x<d \text {, }
\end{aligned}
$$

$$
\begin{gathered}
\tilde{g}(x)=\left\{g(x)-K F_{1}(a) \ln \left|\frac{x-a}{x+a}\right|-K F_{2}(c) \ln \left|\frac{x-c}{x+c}\right|\right\} \text { for } \\
x \in(a, b) \cup(c, d),
\end{gathered}
$$

along with the end conditions that

and

$$
\lambda_{1}(x) \approx\left\{\begin{array}{lll}
O\left(|x-a|^{-1 / 2}\right) & \text { as } & x \rightarrow a \\
O\left(|x-b|^{-1 / 2}\right) & \text { as } & x \rightarrow b
\end{array}\right.
$$

$$
\lambda_{2}(x) \approx\left\{\begin{array}{lll}
O\left(|x-c|^{-1 / 2}\right) & \text { as } & x \rightarrow c \\
O\left(|x-d|^{-1 / 2}\right) & \text { as } & x \rightarrow d .
\end{array}\right.
$$

The solution of the integral equation (2.24) along with the end conditions (2.27) and (2.28) can be derived by using an analysis similar to the one available in the paper of Chakrabarti and George [5], with appropriate modifications (see Appendix) and we find that

$$
\begin{aligned}
\lambda_{1}(x)=\frac{-2\left(A_{1} x^{2}+B_{1}\right) x}{R_{1}(x) \hat{R}_{2}(x)}-\frac{2 x}{\pi^{2}} & {\left[\int_{a}^{b} \frac{\tilde{g}_{1}(t) R_{1}(t) \hat{R}_{2}(t) \mathrm{d} t}{R_{1}(x) \hat{R}_{2}(x)\left(t^{2}-x^{2}\right)}\right.} \\
& \left.-\int_{c}^{d} \frac{\hat{R}_{1}(t) R_{2}(t) \tilde{g}_{2}(t) \mathrm{d} t}{R_{1}(x) \hat{R}_{2}(x)\left(t^{2}-x^{2}\right)}\right] \text { for } a<x<b
\end{aligned}
$$

and

$$
\begin{aligned}
i_{2}(x)=\frac{2\left(A_{1} x^{2}+B_{1}\right) x}{\hat{R}_{1}(x) R_{2}(x)}+\frac{2 x}{\pi^{2}} & {\left[\int_{a}^{b} \frac{\tilde{g}_{1}(t) \hat{R}_{1}(t) R_{2}(t) \mathrm{d} t}{\hat{R}_{1}(x) R_{2}(x)\left(t^{2}-x^{2}\right)}\right.} \\
& \left.-\int_{c}^{d} \frac{\hat{R}_{1}(t) R_{2}(t) \tilde{g}_{2}(t) \mathrm{d} t}{\hat{R}_{1}(x) R_{2}(x)\left(t^{2}-x^{2}\right)}\right] \text { for } c<x<d,
\end{aligned}
$$

where $A_{1}, B_{1}$ are arbitrary constants,

$$
\begin{aligned}
& \tilde{g}_{1}(x)=\tilde{g}(x) \text { for } x \in(a, b) \text { and } \\
& \tilde{g}_{2}(x)=\tilde{g}(x) \text { for } x \in(c, d) \text {, } \\
& R_{1}(x)=\sqrt{\left(x^{2}-a^{2}\right)\left(b^{2}-x^{2}\right)} \text { for } a<x<b \text {, } \\
& \hat{R}_{1}(x)=\left\{\begin{array}{lll}
\sqrt{\left(x^{2}-a^{2}\right)\left(x^{2}-b^{2}\right)} & \text { for } x>b, \\
\sqrt{\left(a^{2}-x^{2}\right)\left(b^{2}-x^{2}\right)} & \text { for } & 0<x<a,
\end{array}\right. \\
& R_{2}(x)=\sqrt{\left(x^{2}-c^{2}\right)\left(d^{2}-x^{2}\right)} \text { for } c<x<d \text {, } \\
& \hat{R}_{2}(x)=\left\{\begin{array}{lll}
\sqrt{\left(d^{2}-x^{2}\right)\left(c^{2}-x^{2}\right)} & \text { for } x<c \\
\sqrt{\left(x^{2}-c^{2}\right)\left(x^{2}-d^{2}\right)} & \text { for } x>d .
\end{array}\right.
\end{aligned}
$$


We then use the following results to simplify the expressions for $\lambda_{1}(x)$ and $\lambda_{2}(x)$ :

$$
\int_{a}^{b} R_{1}(t) \hat{R}_{2}(t) \ln \left|\frac{t-p}{t+p}\right| \frac{\mathrm{d} t}{t^{2}-x^{2}}=\int_{0}^{p} \frac{G_{1}(x, \xi) \mathrm{d} \xi}{\xi^{2}-x^{2}},
$$

where $p=a$ or $c$, and

$$
\begin{aligned}
& G_{1}(x, \xi)=2\left[\int_{a}^{b} t R_{1}(t) \hat{R}_{2}(t)\left\{\frac{1}{t^{2}-x^{2}}+\frac{1}{\xi^{2}-t^{2}}\right\} \mathrm{d} t\right] \\
& \text { for } x \in(a, b) \text { or } x \in(c, d) \\
& \int_{a}^{b} \frac{R_{1}(t) \hat{R}_{2}(t) g_{1}(t) \mathrm{d} t}{R_{1}(x) \hat{R}_{2}(x)\left(t^{2}-x^{2}\right)}=\frac{C_{3}}{R_{1}(x) \hat{R}_{2}(x)}+\frac{\Delta_{1}(x)}{\hat{R}_{2}(x)} \int_{a}^{b} \frac{\hat{R}_{2}(t)}{\Delta_{1}(t)} \frac{g_{1}(t) \mathrm{d} t}{\left(t^{2}-x^{2}\right)}
\end{aligned}
$$$$
\text { for } a<x<b,
$$

where

and

$$
C_{3}=\int_{a}^{b} \frac{\hat{R}_{2}(t)}{\Delta_{1}(t)} g_{1}(t) \mathrm{d} t
$$

$$
\begin{gathered}
\Delta_{1}(x)=\sqrt{\frac{x^{2}-a^{2}}{b^{2}-x^{2}}} \text { for } a<x<b, \\
\int_{c}^{d} \frac{\hat{R}_{1}(t) R_{2}(t) g_{2}(t) \mathrm{d} t}{R_{1}(x) \hat{R}_{2}(x)\left(t^{2}-x^{2}\right)}=\frac{C_{4}}{R_{1}(x) \hat{R}_{2}(x)}-\frac{\Delta_{2}(x)}{\hat{R}_{1}(x)} \int_{c}^{d} \frac{\hat{R}_{1}(t)}{\Delta_{2}(t)} \frac{g_{2}(t) \mathrm{d} t}{\left(t^{2}-x^{2}\right)}, \quad a<x<b,
\end{gathered}
$$

where

and

$$
C_{4}=\int_{c}^{d} \frac{\hat{R}_{1}(t)}{\Delta_{2}(t)} g_{2}(t) \mathrm{d} t
$$

$$
\begin{aligned}
\Delta_{2}(x) & =\sqrt{\frac{c^{2}-x^{2}}{d^{2}-x^{2}}}, \quad a<x<b, \\
\int_{c}^{d} R_{2}(t) \hat{R}_{1}(t) \ln \left|\frac{t-q}{t+q}\right| \frac{\mathrm{d} t}{t^{2}-x^{2}} & =\int_{0}^{a} \frac{G_{2}(x, \xi) \mathrm{d} \xi}{\xi^{2}-x^{2}},
\end{aligned}
$$

where $q=a$ or $q=c$ and

$$
G_{2}(x, \xi)=2\left[\int_{c}^{d} t \hat{R}_{1}(t) R_{2}(t)\left\{\frac{1}{t^{2}-x^{2}}+\frac{1}{\xi^{2}-t^{2}}\right\} \mathrm{d} t\right]
$$

for $x \in(a, b)$ or for $x \in(c, d)$.

$$
\begin{array}{r}
\int_{a}^{b} \frac{R_{1}(t) \hat{R}_{2}(t) g_{1}(t) \mathrm{d} t}{R_{2}(x) \hat{R}_{1}(x)\left(t^{2}-x^{2}\right)}=\frac{C_{3}}{R_{2}(x) \hat{R}_{1}(x)}+\frac{\Delta_{3}(x)}{R_{2}(x)} \int_{a}^{b} \frac{\hat{R}_{2}(t)}{\Delta_{3}(t)\left(t^{2}-x^{2}\right)} \\
\text { for } c<x<d,
\end{array}
$$

where $C_{3}$ is the same as defined in (R7) and 


$$
\begin{gathered}
\Delta_{3}(x)=\sqrt{\frac{x^{2}-a^{2}}{x^{2}-b^{2}}} \text { for } c<x<d, \\
\int_{c}^{d} \frac{\hat{R}_{1}(t) R_{2}(t) g_{2}(t) \mathrm{d} t}{R_{2}(x) \hat{R}_{1}(x)\left(t^{2}-x^{2}\right)}=\frac{C_{4}}{R_{2}(x) \hat{R}_{1}(x)}-\frac{\Delta_{4}(x)}{\hat{R}_{1}(x)} \int_{c}^{d} \frac{\hat{R}_{1}(t)}{\Delta_{4}(t)} \frac{g_{2}(t) \mathrm{d} t}{\left(t^{2}-x^{2}\right)} \\
\text { for } c<x<d,
\end{gathered}
$$

where $C_{4}$ is the same as defined in (R8) and

$$
\Delta_{4}(x)=\sqrt{\frac{x^{2}-c^{2}}{d^{2}-x^{2}}} \text { for } c<x<d .
$$

Using the results (R6) to (R11), we obtain

and

$$
\lambda_{1}(x)=\frac{-2\left[A_{1} x^{2}+Q_{1}+H_{1}(x)\right] x}{R_{1}(x) \hat{R}_{2}(x)}-\frac{2 x}{\pi^{2}} P_{1}(x) \text { for } a<x<b
$$

where

$$
\lambda_{2}(x)=\frac{2\left[A_{1} x^{2}+Q_{1}+H_{1}(x)\right] x}{\hat{R}_{1}(x) R_{2}(x)}+\frac{2 x}{\pi^{2}} \rho_{2}(x) \text { for } c<x<d,
$$

$$
\begin{aligned}
Q_{1}= & B_{1}+\frac{C_{3}-C_{4}}{\pi^{2}} \\
H_{1}(x)= & \frac{K}{\pi^{2}}\left\{F_{1}(a) \int_{0}^{a} \frac{\left[G_{2}(x, \xi)-G_{1}(x, \xi)\right] \mathrm{d} \xi}{\xi^{2}-x^{2}}\right. \\
& \left.+F_{2}(c) \int_{0}^{c} \frac{\left[G_{2}(x, \xi)-G_{1}(x, \xi)\right] \mathrm{d} \xi}{\xi^{2}-x^{2}}\right\}, \\
P_{1}(x)= & \frac{\Delta_{1}(x)}{\hat{R}_{2}(x)} \int_{a}^{b} \frac{\hat{R}_{2}(t) g_{1}(t) \mathrm{d} t}{\Delta_{1}(t)\left(t^{2}-x^{2}\right)}+\frac{\Delta_{2}(x)}{R_{1}(x)} \int_{c}^{d} \frac{\hat{R}_{1}(t) g_{2}(t) \mathrm{d} t}{\Delta_{2}(t)\left(t^{2}-x^{2}\right)} \text { for } a<x<b
\end{aligned}
$$

and

$$
P_{2}(x)=\frac{\Delta_{3}(x)}{R_{2}(x)} \int_{a}^{b} \frac{\hat{R}_{2}(t) g_{1}(t) \mathrm{d} t}{\Delta_{3}(t)\left(t^{2}-x^{2}\right)}-\frac{\Delta_{4}(x)}{\hat{R}_{1}(x)} \int_{c}^{d} \frac{\hat{R}_{1}(t) g_{2}(t) \mathrm{d} t}{\Delta_{4}(t)\left(t^{2}-x^{2}\right)} \text { for } c<x<d
$$

Now using the relations (2.25), we find that

and

$$
\begin{aligned}
& \mathrm{e}^{K a} F_{1}(a)=\int_{a}^{b} \mathrm{e}^{K u} \lambda_{1}(u) \mathrm{d} u \text { and } \\
& \mathrm{e}^{K c} F_{2}(c)=\int_{c}^{d} \mathrm{e}^{K u} \dot{\lambda}_{2}(u) \mathrm{d} u,
\end{aligned}
$$

$$
\begin{aligned}
& f_{1}(x)=\frac{\mathrm{d}}{\mathrm{d} x}\left[\mathrm{e}^{-K x} \int_{x}^{b} \mathrm{e}^{K u} \lambda_{1}(u) \mathrm{d} u\right], \text { for } a<x<b \text { and } \\
& f_{2}(x)=\frac{\mathrm{d}}{\mathrm{d} x}\left[\mathrm{e}^{-K x} \int_{x}^{d} \mathrm{e}^{K u} \lambda_{2}(u) \mathrm{d} u\right] \text { for } c<x<d,
\end{aligned}
$$

where $\hat{\lambda}_{1}(x)$ and $\lambda_{2}(x)$ are given by the expressions (2.36) and (2.37) respectively. 
The relations (2.42) completely solve (1.1), in this case, if the two relations in (2.41) are utilized to determine the constants $F_{1}(a)$ and $F_{2}(c)$ in terms of $A_{1}$ and $Q_{1}$, which remain arbitrary. Alternative forms of the functions $f_{1}$ and $f_{2}$ can also be derived, as has been done in the case (i), where the solution has been expressed through the relations (2.15) and (2.16).

\section{Conclusion}

A unified approach has been developed to solve the singular integral equations of water wave problems, which involve a union of disjoint finite intervals, at the end points of which the unknown function is required to satisfy square-root type integrable singularities. The cases of single as well as double intervals have been analyzed here in detail. For more number of intervals (greater than two), the method is similar, even though a little more involved.

\section{Appendix}

Solution of (2.24)

Equation (2.24) can be cast into the form

and

$$
T_{1} \lambda_{1}^{*}(\eta)+\tilde{T_{2}} \lambda_{2}^{*}(\eta)=g_{1}^{*}(\eta) \text { for } \eta \in\left(a_{1}, b_{1}\right)
$$

$$
\tilde{T}_{1} \lambda_{1}^{*}(\eta)+T_{2} \lambda_{2}^{*}(\eta)=g_{2}^{*}(\eta) \text { for } \eta \in\left(c_{1}, d_{1}\right)
$$

after using the transformations

$$
\begin{aligned}
& x^{2}=\eta, t^{2}=\xi, \lambda_{1}^{*}(\xi)=\frac{\lambda_{1}(\sqrt{\xi})}{2 \sqrt{\xi}}, \lambda_{2}^{*}(\xi)=\frac{\lambda_{2}(\sqrt{\xi})}{2 \sqrt{\xi}}, a^{2}=a_{1}, b^{2}=b_{1}, c^{2}=c_{1}, \\
& d^{2}=d_{1}, g_{1}^{*}(\eta)=\frac{\tilde{g}_{1}(\sqrt{\eta})}{2 \sqrt{\eta}}, \eta \in\left(a_{1}, b_{1}\right), g_{2}^{*}(\eta)=\frac{\tilde{g}_{2}(\sqrt{\eta})}{2 \sqrt{\eta}}, \eta \in\left(c_{1}, d_{1}\right)
\end{aligned}
$$

and employing the operators $T_{1}, T_{2}, \widetilde{T}_{1}$ and $\tilde{T}_{2}$ as defined by the relations

$$
\begin{aligned}
& T_{1} \lambda_{1}^{*}(\eta)=\int_{a_{1}}^{b_{1}} \frac{\lambda_{1}^{*}(\xi) \mathrm{d} \xi}{\xi-\eta} \text { for } \eta \in\left(a_{1}, b_{1}\right), \\
& T_{2} \lambda_{2}^{*}(\eta)=\int_{c_{1}}^{d_{1}} \frac{\lambda_{2}^{*}(\xi) \mathrm{d} \xi}{\xi-\eta} \text { for } \eta \in\left(c_{1}, d_{1}\right), \\
& \tilde{T}_{1} \lambda_{1}^{*}(\eta)=\int_{a_{1}}^{b_{1}} \frac{\lambda_{1}^{*}(\xi) \mathrm{d} \xi}{\xi-\eta} \text { for } \eta \notin\left(a_{1}, b_{1}\right), \\
& \tilde{T}_{2} \lambda_{2}^{*}(\eta)=\int_{c_{1}}^{d_{1}} \frac{\lambda_{2}^{*}(\xi) \mathrm{d} \xi}{\xi-\eta} \text { for } \eta \notin\left(c_{1}, d_{1}\right) .
\end{aligned}
$$

We then define the inverse operators $T_{1}^{-1}$ and $T_{2}^{-1}$ as given by

$$
\begin{aligned}
& T_{1}^{-1} \lambda_{1}^{*}(\eta)=\frac{D_{1}}{\Delta_{3}(\eta)}-\frac{T_{1}\left(\Delta_{3}(\eta) \lambda_{1}^{*}(\eta)\right)}{\pi^{2} \Delta_{3}(\eta)} \text { for } \eta \in\left(a_{1}, b_{1}\right) \text { and } \\
& T_{2}^{-1} \lambda_{2}^{*}(\eta)=\frac{D_{2}}{\Delta_{4}(\eta)}-\frac{T_{2}\left(\Delta_{4}(\eta) \lambda_{2}^{*}(\eta)\right)}{\pi^{2} \Delta_{4}(\eta)} \text { for } \eta \in\left(c_{1}, d_{1}\right),
\end{aligned}
$$


where $D_{1}$ and $D_{2}$ are two arbitrary constants and

$$
\begin{aligned}
& \Delta_{3}(\eta)=\sqrt{\left(\eta-a_{1}\right)\left(b_{1}-\eta\right)} \text { for } \eta \in\left(a_{1}, b_{1}\right) \text { and } \\
& \Delta_{4}(\eta)=\sqrt{\left(\eta-c_{1}\right)\left(d_{1}-\eta\right)} \text { for } \eta \in\left(c_{1}, d_{1}\right) .
\end{aligned}
$$

We easily derive the following results $(\tilde{\mathrm{R}} 1-\tilde{\mathrm{R}} 8)$, by using standard methods.

$$
\begin{aligned}
& T_{1}\left(\Delta_{3}(\eta)\right)=\pi\left[-\eta+\frac{a_{1}+b_{1}}{2}\right] \text { for } \eta \in\left(a_{1}, b_{1}\right), \\
& T_{1}\left(\Delta_{3}(\eta) \tilde{T}_{2} \lambda_{2}^{*}(\eta)\right)=\pi \int_{c_{1}}^{d_{1}} \lambda_{2}^{*}(\eta) \mathrm{d} \eta-\pi \tilde{T}_{2}\left(\hat{\Delta}_{3}(\eta) \lambda_{2}^{*}(\eta)\right) \text { for } a_{1}<\eta<b_{1},
\end{aligned}
$$

where $\hat{\Delta}_{3}(\eta)=\sqrt{\left(\eta-a_{1}\right)\left(\eta-b_{1}\right)}$ for $\eta \notin\left(a_{1}, b_{1}\right)$,

$$
\begin{aligned}
& \tilde{T}_{1}\left(\frac{1}{\Delta_{3}(\eta)} \tilde{T}_{2}\left(\hat{\Delta}_{3}(\eta) \lambda_{2}^{*}(\eta)\right)\right)=\pi T_{2}\left(\lambda_{2}^{*}(\eta)\right)-\frac{\pi}{\hat{\Delta}_{3}(\eta)} T_{2}\left(\hat{\Delta}_{3}(\eta) \hat{\lambda}_{2}^{*}(\eta)\right) \\
& \tilde{T}_{1}\left(\frac{1}{\Delta_{3}(\eta)} T_{1}\left(\Delta_{1}(\eta) g_{1}^{*}(\eta)\right)\right)=-\frac{\pi}{\hat{\Delta}_{3}(\eta)} \tilde{T}_{1}\left(\Delta_{3}(\eta) g_{1}^{*}(\eta)\right) \text { for } \eta \in\left(c_{1}, d_{1}\right) \\
& \left.T_{2}\left(\Delta_{4}(\eta) \tilde{T}_{1}\left(\Delta_{3}(\eta) g_{1}^{*}(\eta)\right)\right)=\pi \int_{a_{1}}^{b_{1}} \Delta_{1}\right)
\end{aligned}
$$

where $\hat{\Delta}_{4}(\eta)=\sqrt{\left(c_{1}-\eta\right)\left(d_{1}-\eta\right)}$

$$
\begin{aligned}
& \tilde{T}_{2}\left(\frac{1}{\Delta_{4}(\eta)} \tilde{T}_{1}\left(\Delta_{3}(\eta) \hat{\Delta}_{4}(\eta) g_{1}(\eta)\right)\right)= \frac{\pi}{\Delta_{4}(\eta)} T_{1}\left(\Delta_{3}(\eta) \hat{\Delta}_{4}(\eta) g_{1}(\eta)\right) \\
&-\pi T_{1}\left(\Delta_{3}(\eta) g_{1}(\eta)\right) \\
& \tilde{T}_{2}\left(\frac{1}{\Delta_{4}(\eta)} T_{2}\left(\hat{\Delta}_{3}(\eta) \Delta_{4}(\eta) g_{2}^{*}(\eta)\right)\right)= \frac{\pi}{\Delta_{4}(\eta)} \tilde{T}_{2}\left(\Delta_{4}(\eta) \hat{\Delta}_{3}(\eta) g_{2}^{*}(\eta)\right) \\
& \tilde{T}_{2}\left(\frac{\eta}{\widehat{\Delta}_{4}(\eta)}\right)=\pi\left(1+\frac{\eta}{\hat{\Delta}_{4}(\eta)}\right) \text { for } \eta \in\left(a_{1}, b_{1}\right) .
\end{aligned}
$$

Applying the operator $T_{1}^{-1}$ to eq. (A1) and using the result ( $(\tilde{\mathrm{R}} 2)$, we obtain

$$
\dot{\lambda}_{1}^{*}(\eta)=\frac{A_{1}}{\Delta_{3}(\eta)}-\frac{\pi \tilde{T}_{2}\left(\Delta_{3}(\eta)\right)+T_{1}\left(\Delta_{3}(\eta) g_{1}^{*}(\eta)\right)}{\pi^{2} \Delta_{3}(\eta)}
$$

where $A_{1}=D_{1}+(1 / \pi) \int_{c_{1}}^{d_{1}} \hat{\lambda}_{2}^{*}(s) \mathrm{d} s$ is an arbitrary constant. form

Using the expression (A6) along with the results ( $(\tilde{R} 3)$ and ( $(\tilde{R} 4)$, we can rewrite (A2) in the

$$
T_{2}\left(\hat{\Delta}_{3}(\eta) \lambda_{2}^{*}(\eta)\right)=A_{1} \pi-\frac{1}{\pi} \tilde{T}_{1}\left(\Delta_{3}(\eta) g_{1}^{*}(\eta)\right)+\hat{\Delta}_{3}(\eta) g_{2}^{*}(\eta) \text { for } \eta \in\left(c_{1}, d_{1}\right)
$$


Applying the inverse operator $T_{2}^{-1}$ to eq. (A7), we obtain, after using the results $(\tilde{\mathbf{R}} 1),(\tilde{\mathbf{R}} 5)$, that

$$
\begin{aligned}
& \lambda_{2}^{*}(\eta)=\frac{A_{1} \eta+B_{1}}{\Delta_{4}(\eta) \hat{\Delta}_{3}(\eta)}-\frac{T_{2}\left(\Delta_{4}(\eta) \hat{\Delta}_{3}(\eta) g_{2}^{*}(\eta)\right)-\tilde{T}_{1}\left(\Delta_{3}(\eta) \hat{\Delta}_{4}(\eta) g_{1}^{*}(\eta)\right)}{\pi^{2} \hat{\Delta}_{3}(\eta) \Delta_{4}(\eta)} \\
& \text { for } \eta \in\left(c_{1}, d_{1}\right) \text {, }
\end{aligned}
$$

where $B_{1}\left(=D_{2}-\left(A_{1} / 2\right)\left(c_{1}+d_{1}\right)+\pi^{-2} \int_{a_{1}}^{b_{1}} \Delta_{3}(\eta) g_{1}^{*}(\eta) \mathrm{d} \eta\right)$ is an arbitrary constant.

The relations (A6) and (A8), after using the results $(\tilde{\mathbf{R}} 5)$ to $(\tilde{\mathbf{R}} 8)$ ultimately give

$$
\begin{array}{r}
\lambda_{1}^{*}(\eta)=\frac{-A_{1} \eta-B_{1}}{\Delta_{3}(\eta) \hat{\Delta}_{4}(\eta)}-\frac{T_{1}\left(\Delta_{3}(\eta) \hat{\Delta}_{4}(\eta) g_{1}^{*}(\eta)\right)-\tilde{T}_{2}\left(\Delta_{4}(\eta) \hat{\Delta}_{3}(\eta) g_{2}^{*}(\eta)\right)}{\pi^{2} \hat{\Delta}_{4}(\eta) \Delta_{3}(\eta)} \\
\text { for } a_{1}<\eta<b_{1} .
\end{array}
$$

Going back to the original variables $x, t, \lambda_{1}$ and $\lambda_{2}$, we get the results (2.29) and (2.30) used in the paper.

\section{Acknowledgements}

The authors are thankful to the referee for his comments and suggestions to improve the presentation of the paper. TS acknowledges the University Grants Commission, New Delhi, for the financial support as a research student of Indian Institute of Science. TS also acknowledges the partial support of CSIR, New Delhi during the period when the paper was revised.

\section{References}

[1] Muskhelishvili N I, Singular integral equation (Noordhoof, Holland) (1963)

[2] Ursell F, The effect of a fixed vertical barrier on surface waves in deep water, Proc. Camb. Phil. Soc. 43 (1947) 374-382

[3] Mandal B N, A note on the diffraction of water waves by a vertical wall with a gap, Arch. Mech. 39 (1987) 269-273

[4] Gakhov F D, Boundary value problems (Dover Publications: New York) (1990)

[5] Chakrabarti $A$ and George A J, Solution of a singular integral equation involving two intervals arising in the theory of water waves, Appl. Math. Lett. 7(5) (1994) 43-47

[6] Banerjea $S$ and Mandal B N, On a singular integral equation with logarithmic and Cauchy kernel, Int'l J. Math. Educ. Sci. Technol. 26(2) (1995) 267-313 